\title{
Benchmarking subcellular localization and variant tolerance predictors on membrane proteins
}

Tommaso Orioli ${ }^{1,2}$ and Mauno Vihinen ${ }^{2^{*}}$

From Varl-COSI 2018: identification and annotation of genetic variants in the context of structure, function, and disease Chicago, IL, USA. 08 July 2018

\begin{abstract}
Background: Membrane proteins constitute up to 30\% of the human proteome. These proteins have special properties because the transmembrane segments are embedded into lipid bilayer while extramembranous parts are in different environments. Membrane proteins have several functions and are involved in numerous diseases. A large number of prediction methods have been introduced to predict protein subcellular localization as well as the tolerance or pathogenicity of amino acid substitutions.

Results: We tested the performance of 22 tolerance predictors by collecting information on membrane proteins and variants in them. The analysis indicated that the best tools had similar prediction performance on transmembrane, inside and outside regions of transmembrane proteins and comparable to overall prediction performances for all types of proteins. PON-P2 had the highest performance followed by REVEL, MetaSVM and VEST3. Further, we tested with the high quality dataset also the performance of seven subcellular localization predictors on membrane proteins. We assessed separately the performance for single pass and multi pass membrane proteins. Predictions for multi pass proteins were more reliable than those for single pass proteins.

Conclusions: The predictors for variant effects had better performance than subcellular localization tools. The best tolerance predictors are highly reliable. As there are large differences in the performances of tools, end-users have to be cautious in method selection.
\end{abstract}

Keywords: Membrane protein, Benchmark, Benchmarking, Variation interpretation, Method performance, Disease-causing variant, Mutation

\section{Background}

Cells and compartments within them are surrounded by membranes composed of lipids having two opposed layers of amphipathic molecules. The bilayers contain in addition to lipids many other molecules, among them proteins that have numerous functions. Membrane proteins (MPs) are crucial for membrane stability and cellular functions due to their ability to communicate with the environment outside and inside of membranes. $25-30 \%$ of human proteins have

\footnotetext{
* Correspondence: mauno.vihinen@med.lu.se

${ }^{2}$ Department of Experimental Medical Science, BMC B13, Lund University,

SE-22184 Lund, Sweden

Full list of author information is available at the end of the article
}

been estimated to be transmembrane proteins (TMPs) [1]. These proteins are important in many ways, for example $60 \%$ of current pharmaceutical drug targets are MPs $[2,3]$. Organelles within cells, such as mitochondria, endoplasmic reticulum (ER), and Golgi apparatus, are maintained by their membranes that carry specific MPs.

MPs can be classified in many ways. Structure based classification is widely used [4]. It discriminates the MPs depending on how they interact with the lipid membrane. Type I membrane proteins, also known as single pass TMPs have extracellular (or luminal) N-terminus and cytoplasmic C-terminus, while type II TMPs have the opposite: extracellular (or luminal) C-terminus and cytoplasmic $\mathrm{N}$ -

(c) The Author(s). 2019 Open Access This article is distributed under the terms of the Creative Commons Attribution 4.0 International License (http://creativecommons.org/licenses/by/4.0/), which permits unrestricted use, distribution, and 
terminus. These two types of MPs represent about half of the human membrane proteome [5]. Multi pass TMPs trespass through the membrane several times. Lipid chainanchored membrane proteins are located on the surface of the cell membrane and are covalently attached to lipids and can be located on both sides of the membrane. Lipid attaches at or near the C-terminus of the protein and plays a crucial role in the protein function. GPI-anchored MPs are attached via glycosylphosphatidylinositol (GPI) group. Peripheral membrane proteins are bound to the membrane indirectly by non-covalent interaction with other membrane proteins. Therefore they are not considered as proper MPs.

We concentrated on TMPs of types I and II and multi pass proteins as they are permanently attached to and span through the membrane.

Besides the topology, MPs can be classified as $\alpha$-helix and $\beta$-barrel proteins. $\alpha$-Helical MPs are organized as anti-parallel bundles, which are typically tilted with respect to the membrane by $25^{\circ}[6]$. We focused on $\alpha-$ helical MPs, since in human, $\beta$-barrel MPs appear only in the outer membranes of mitochondria.

MPs are involved in numerous functions. Information has been collected to databases such as Orientations of Proteins in Membranes (OPM [7]), Topology Database of Transmembrane Proteins (TOPDB [8]) and Human Transmembrane Proteome (HTP [1]), in addition to more general databases including Protein Data Bank (PDB [9]) and UniProtKB [10].

In an effort to classify human membrane proteome, 6684 non-redundant genes were clustered to 234 protein families or groups, and four major functional categories [5]. Receptors mediate cellular response upon binding to a ligand. The most representative groups are G-protein coupled receptors, receptor-type kinases, receptors of immunoglobulin superfamily and scavenger receptors. Transporters move ions and molecules from the extracellular environment into cells, and vice versa, by utilizing electrochemical gradients or through chemical reactions. They are grouped as channels, solute carriers and active transporters. Enzymes catalyze different reactions. Miscellaneous group contains MPs that have other functions such as ligands, or structural or adhesion proteins.

The Transporter Classification Database (TCDB) is an International Union of Biochemistry and Molecular Biology (IUBMB)-approved classification system groups membrane transport proteins to seven classes: channels and pores, electrochemical potential-driven transporters, primary active transporters, group translocators, transport electron carriers, accessory factors involved in transport, and incompletely characterized transport systems [11]. According to the Enzyme Commission number classification (EC number) there are seven main classes: oxidoreductases, transferases, hydrolases, lyases, isomerases, ligases and translocases [12].
We retrieved high-quality datasets of TMPs and used them to benchmark how good protein localization predictors are since the existing benchmark studies are based on smaller and less reliable data. We collected also a dataset of disease-related variants in these TMPs and tested the performance of protein pathogenicity/tolerance predictors including the three regions in these proteins: inside and outside as well as within the membranes. This is to our knowledge the first study on the membrane protein variant prediction performance.

\section{Results and discussion}

Our aim was to evaluate the performance of variant predictors on TMPs. For that purpose we collected datasets of both MPs and variants in them. The datasets were then used to benchmark predictors on two major characteristics of membrane proteins, namely subcellular localization and variant tolerance. The exact number of TMPs is not known, but various estimates have been presented. According to the data in HTP, the human proteome contains 25-30\% of MPs. We collected information for both experimentally defined and predicted MPs.

Human Protein Atlas is a long term project for analysis of human proteins. Until now, they provide detailed experimental information for the subcellular localizations of 12,073 proteins. The data contain reliability scores. We obtained two datasets from HPA. MP1289 contains 1289 proteins reliability of which were validated, supported or approved.

The test sets are summarized in Table 1. In order to calculate performance indices, the negative sets were normalized to have the same number of proteins as the positive set.

Additional file 1 Table S1 shows that $42 \%$ of the proteins are found in one location (542 out of 1289), the majority are multi location proteins (MLPs). Out of the 747 MLPs, $83.0 \%$ (620) were found in two locations, $16.7 \%$ (125) in three locations and $0.3 \%$ (2) in four locations. We retrieved data for 124 validated (9.6\%), 439 supported (34.1\%) and 726 approved (56.3\%) proteins.

Table 1 Numbers of proteins in test sets

\begin{tabular}{|c|c|c|c|c|c|c|}
\hline & \multicolumn{2}{|l|}{ MP1289 } & \multicolumn{2}{|l|}{ MP508 } & \multicolumn{2}{|l|}{ mpHTP } \\
\hline & Positive & Negative & Positive & Negative & Positive & Negative \\
\hline BUSCA & 1040 & 986 & 393 & 361 & 4550 & 4362 \\
\hline CELLO & 1223 & 1180 & 474 & 449 & 5045 & 5194 \\
\hline DeepLoc1.0 & 1285 & 931 & 505 & 337 & 4669 & 4629 \\
\hline LocTree3 & 1286 & 1270 & 507 & 497 & 5358 & 5289 \\
\hline MultiLoc2 & 1106 & 1193 & 408 & 464 & 4654 & 4999 \\
\hline SubCons & 852 & 744 & 309 & 292 & 4055 & 3882 \\
\hline Wolf PSORT & 1289 & 1288 & 508 & 507 & 5414 & 5362 \\
\hline
\end{tabular}


For a subset of even more reliable data we excluded those with annotation approved and obtained 508 proteins called MP508. The percentage of MLPs is smaller in this set, $50.6 \%$ (Additional file 1: Table S1). Out of 257 MLPs, $83.7 \%$ (215) were found in two different locations and $16.3 \%$ (42) in three different locations. The dataset contains 124 validated $(24.4 \%)$ and 384 supported $(75.6 \%)$ plasma membrane and nuclear MPs. The third dataset, called mpHTP, is the largest one and contains localizations of 5422 human proteins.

\section{Performance of subcellular localization predictors}

We searched from literature and internet for protein subcellular localization predictors and found several ones. We included to our analysis seven methods that were available as web service or downloadable standalone version and allowed submission of large number of sequences. The methods are listed in Table 2. We could not test some other tools, because they did not support multiple sequence submission, including MemPype [20], HSLPred [21] and Iloc-Hum [22], or because problems with availability of the server, i.e. SherLoc2 [23]. We compared the predicted localizations to experimentally verified results and calculated six performance measures. Proteins used for training the methods were excluded when these details were available. We were not able to retrieve the training set of Wolf PSORT. Protein sequences were matched based on UniProt ID match and by similarity obtained with BLASTP [24].

Results in Table 1 indicate the numbers of predicted proteins for the 7 tested tools. None of the tools was able to predict all the cases in the three positive and negative test sets, however, Wolf PSORT comes very close.

Tables 3, 4 and 5 contain performance assessments for MP1289, MP508 and mpHTP, respectively. The first two datasets contain plasma membrane and nuclear membrane proteins, whereas the mpHTP contains TMPs from all sub compartments. DeepLoc1.0 and LocTree3 predict whether a protein is a MP or a cytoplasmic protein, besides the subcellular localization. Some predictors return a single result (BUSCA, DeepLoc1.0, LocTree3,
SubCons) with a probability, whereas CELLO, MultiLoc2 and Wolf PSORT return the probability distribution associated to each location. In the latter case, we considered the possibility of having a double localization when there was not one with a predominant probability (i.e. $>0.50$ ).

The performance of all methods is very low on both MP1289 and MP508 (Tables 3 and 4). The MCC values range from 0.11 (MultiLoc2) to 0.35 (SubCons) in the MP1289 and from 0.20 (MultiLoc2) to 0.47 (SubCons) for MP508. In addition to having low overall performance, the tools are very biased, especially sensitivity is very low while specificity has the highest score among the tested ones for all the methods and on both the datasets.

All the measures are somewhat better for MP508. The tools are largely under predicting MPs. When just about one out of three or four of the real MPs are predicted correct, the overall performance remains low (Tables 3 and 4) . Consequently, the number of false negatives is very high. Specificity is higher, but that is because the number of false negatives is two times higher than that for the true positives. It can be concluded that if these tools predict a protein to be an MP it is highly likely true, the problem is that they miss $60 \%$ or more of the cases.

The performance scores are better for the mpHTP set. The MCC ranges from 0.85 for DeepLoc1.0 to 0.34 for MutliLoc2. In this case also the sensitivity is clearly better, from 0.60 to 0.93 , except for MultiLoc2, which has a value of 0.24 . DeepLoc1.0 has the best and MultiLoc2 the lowest OPM on all the datasets. The performances are contradictory for the other methods, each of BUSCA, LocTree, SubCons and Wolf PSORT showing good performance on some datasets. BUSCA, DeepLoc and LocTree3 can predict either the subcellular localization or whether a protein is a MP. On the mpHTP data, DeepLoc1.0 is clearly the best balanced method, since values for sensitivity, specificity, PPV and NPV span from 0.91 to 0.94 .

As seen above, the results are very sensitive for the composition of the dataset. The majority of the proteins in MP1289 and MP508 are MLPs, 57.7 and 50.6\% respectively. When we filtered out all the MLPs from the

Table 2 Subcellular localization predictors

\begin{tabular}{llll}
\hline Method & Description & URL & Reference \\
\hline BUSCA & Metapredictor for localization-related protein features & http://busca.biocomp.unibo.it/ & [13] \\
CELLO & Two-layer SVM & http://cello.life.nctu.edu.tw/ & http://www.cbs.dtu.dk/services/DeepLoc/ $\quad[15]$ \\
DeepLoc & Deep neural network & https://rostlab.org/services/loctree2/ & [14] \\
LOCTREE3 & SVM & https://github.com/KohlbacherLab/ & MultiLoc2/tree/master/MultiLoc2 \\
MultiLoc2 & SVM & http://subcons.bioinfo.se/ & [17] \\
SubCons & RF & https://wolfpsort.hgc.jp/ & [18] \\
Wolf & Converts amino acid sequences into numerical vectors that are grouped with & \\
PSORT & a weighted k-nearest neighbor classifier & & [19] \\
\hline
\end{tabular}


Table 3 Performance of subcellular localization predictors on MP1289

\begin{tabular}{|c|c|c|c|c|c|c|c|}
\hline & BUSCA & CELLO & DeepLoc 1.0 & LocTree3 & MultiLoc2 & SubCons & WolfPSORT \\
\hline $\mathrm{TP}$ & 316 & 348 & 447 & 344 & 108 & 242 & 516 \\
\hline FP & 112 & 158 & 102 & 75 & 50 & 42 & 218 \\
\hline TN & 763 & 1073 & 987 & 1203 & 1144 & 1152 & 1071 \\
\hline FN & 724 & 875 & 838 & 942 & 998 & 610 & 773 \\
\hline Sensitivity & 0.36 & 0.28 & 0.41 & 0.27 & 0.09 & 0.2 & 0.4 \\
\hline Specificity & 0.87 & 0.87 & 0.91 & 0.94 & 0.96 & 0.96 & 0.83 \\
\hline PPV & 0.74 & 0.69 & 0.81 & 0.82 & 0.68 & 0.85 & 0.7 \\
\hline NPV & 0.51 & 0.55 & 0.54 & 0.56 & 0.53 & 0.65 & 0.58 \\
\hline ACC & 0.56 & 0.58 & 0.6 & 0.6 & 0.54 & 0.68 & 0.62 \\
\hline MCC & 0.21 & 0.19 & 0.3 & 0.28 & 0.11 & 0.35 & 0.26 \\
\hline OPM & 0.23 & 0.21 & 0.28 & 0.26 & 0.18 & 0.3 & 0.25 \\
\hline
\end{tabular}

MP1289 and reassessed the performance, the performances increased, but still the best performing tools (DeepLoc1.0 and Wolf PSORT) had MCC of 0.51 and OPM of 0.43 and 0.42 (Additional file 1: Table S2).

Next, we evaluated the performance of the predictors by dividing the mpHTP dataset into two subsets, those containing one TM region i.e. single pass proteins and those containing $>1 \mathrm{TM}$ region (multi pass). The results indicate that all the predictors are performing better for multi pass proteins (Additional file 1: Table S3). Now, in addition to BUSCA and DeepLoc1.0, CELLO excels with second highest MCC of 0.88 . One explanation for the better detection of multi pass proteins may be that since there are several TM regions the predictors have more chances in detecting them.

\section{Performance of tolerance predictors on variants in MPs}

Tolerance predictors are widely used to investigate outcomes of identified variants. Tens of such methods have been developed. Performances of these methods have been previously tested [25-28], but not specifically for MPs. As the training datasets of machine learning methods (ML) do not contain that many MPs, it was of interest to find how the tools work on membrane proteins. We collected a set of 2058 variants, 747 of which were disease-related and 1311 which had high $(>1 \%$ but $<25 \%)$ minor allele frequency in ExAC and which can be considered as benign.

We tested altogether 22 methods, principles of which are widely different, for a summary see [29]. The methods were run on default parameters. Variants used to train PON-P2 (7016 in TMPs, 3990 deleterious and 3026 neutral) were excluded from tests.

The general performances of all the predictors are summarized in Table 6 and in Fig. 1. Similar to previous assessments on proteins in general, the performances vary widely. The MCC values range from 0.13 for fitCons to 0.87 for PON-P2 and the OPM from 0.18 (fitCons) to 0.82 (PON-P2). Altogether, six methods have the MCC equal or higher than 0.80 , namely PON-P2 (0.87), REVEL (0.83), MetaSVM (0.81), MutPred (0.80), PolyPhen HVAR (0.80) and VEST3 (0.80).

Out of 2058 variants in the dataset, 1934 (94\%) were correctly predicted by the best performing tool (PONP2): 338 out of 367 (92.1\%) in transmembrane region

Table 4 Performance of subcellular localization predictors on MP508

\begin{tabular}{|c|c|c|c|c|c|c|c|}
\hline & BUSCA & CELLO & DeepLoc1.0 & LocTree3 & MultiLoc2 & SubCons & Wolf PSORT \\
\hline$\overline{\mathrm{TP}}$ & 126 & 136 & 218 & 166 & 49 & 110 & 227 \\
\hline FP & 18 & 28 & 20 & 10 & 9 & 6 & 40 \\
\hline TN & 282 & 438 & 350 & 493 & 437 & 440 & 468 \\
\hline FN & 267 & 338 & 287 & 341 & 359 & 199 & 281 \\
\hline Sensitivity & 0.42 & 0.29 & 0.59 & 0.33 & 0.11 & 0.25 & 0.45 \\
\hline Specificity & 0.94 & 0.94 & 0.95 & 0.98 & 0.98 & 0.99 & 0.92 \\
\hline PPV & 0.88 & 0.83 & 0.92 & 0.94 & 0.84 & 0.95 & 0.85 \\
\hline NPV & 0.51 & 0.56 & 0.55 & 0.59 & 0.55 & 0.69 & 0.62 \\
\hline ACC & 0.59 & 0.61 & 0.65 & 0.65 & 0.57 & 0.73 & 0.68 \\
\hline MCC & 0.32 & 0.30 & 0.42 & 0.41 & 0.20 & 0.47 & 0.42 \\
\hline OPM & 0.29 & 0.27 & 0.38 & 0.34 & 0.22 & 0.37 & 0.35 \\
\hline
\end{tabular}


Table 5 Performance of subcellular localization predictors on mpHTP

\begin{tabular}{|c|c|c|c|c|c|c|c|}
\hline & BUSCA & CELLO & DeepLoc1.0 & LocTree3 & MultiLoc2 & SubCons & Wolf PSORT \\
\hline $\mathrm{TP}$ & 3766 & 3101 & 4242 & 3877 & 1187 & 2776 & 4016 \\
\hline FP & 206 & 153 & 257 & 179 & 104 & 103 & 280 \\
\hline TN & 4156 & 5041 & 4372 & 5110 & 4895 & 3779 & 5082 \\
\hline FN & 784 & 1944 & 427 & 1481 & 3467 & 1279 & 1398 \\
\hline Sensitivity & 0.86 & 0.60 & 0.93 & 0.73 & 0.24 & 0.71 & 0.75 \\
\hline Specificity & 0.95 & 0.97 & 0.94 & 0.97 & 0.98 & 0.97 & 0.95 \\
\hline PPV & 0.95 & 0.95 & 0.94 & 0.96 & 0.92 & 0.96 & 0.93 \\
\hline NPV & 0.84 & 0.72 & 0.91 & 0.78 & 0.58 & 0.75 & 0.78 \\
\hline ACC & 0.89 & 0.79 & 0.93 & 0.84 & 0.63 & 0.83 & 0.84 \\
\hline MCC & 0.78 & 0.63 & 0.85 & 0.71 & 0.34 & 0.68 & 0.70 \\
\hline OPM & 0.72 & 0.53 & 0.80 & 0.62 & 0.30 & 0.60 & 0.62 \\
\hline
\end{tabular}

and 1559 out of 1691 (92.2\%) in non-transmembrane region. Of 747 pathogenic and 1311 neutral variants, 699 (93.6\%) and 1235 (94.2\%) were correctly predicted, respectively.

There are no major differences for the three regions in the TMPs, i.e. transmembrane, the inner region and the outer region (Table 7), except for Eigen, Eigen-PC, fitCons, and MutationAssessor when looking at accuracy, MCC and OPM. Twelve of the methods have the best performance for inner membrane variants, 5 methods for outer membrane and 5 methods for transmembrane regions, however the differences are typically very small. Of the 10 best methods, seven perform somewhat better for outer membrane and 3 for transmembrane variants than on the other parts in the MPs. The best methods, in particular PON-P2 and REVEL, are also the most balanced when all the assessment measures are considered. Methods with lower performances, such as CADD,

Table 6 Overall performance of tolerance predictors on MP variants

\begin{tabular}{|c|c|c|c|c|c|c|c|c|c|c|c|}
\hline & TP & FP & $\mathrm{TN}$ & FN & Sensitivity & Specificity & PPV & NPV & ACC & MCC & OPM \\
\hline CADD & 725 & 670 & 641 & 18 & 0.98 & 0.49 & 0.66 & 0.95 & 0.73 & 0.53 & 0.44 \\
\hline DANN & 737 & 1006 & 305 & 6 & 0.99 & 0.23 & 0.56 & 0.97 & 0.61 & 0.35 & 0.30 \\
\hline Eigen & 582 & 253 & 1058 & 161 & 0.78 & 0.81 & 0.80 & 0.79 & 0.80 & 0.59 & 0.50 \\
\hline Eigen-PC & 580 & 266 & 1045 & 163 & 0.78 & 0.80 & 0.79 & 0.78 & 0.79 & 0.58 & 0.49 \\
\hline FATHMM & 556 & 230 & 1053 & 183 & 0.75 & 0.82 & 0.81 & 0.77 & 0.79 & 0.57 & 0.49 \\
\hline FATHMM-MKL & 705 & 434 & 877 & 38 & 0.95 & 0.67 & 0.74 & 0.93 & 0.81 & 0.64 & 0.55 \\
\hline FitCons & 423 & 571 & 740 & 320 & 0.57 & 0.56 & 0.57 & 0.57 & 0.57 & 0.13 & 0.18 \\
\hline GenoCanyon & 605 & 313 & 998 & 138 & 0.81 & 0.76 & 0.77 & 0.80 & 0.79 & 0.58 & 0.49 \\
\hline LRT & 625 & 205 & 904 & 91 & 0.87 & 0.82 & 0.83 & 0.87 & 0.84 & 0.69 & 0.60 \\
\hline M-CAP & 707 & 104 & 152 & 13 & 0.98 & 0.59 & 0.71 & 0.97 & 0.79 & 0.62 & 0.53 \\
\hline MetaLR & 615 & 76 & 1235 & 128 & 0.83 & 0.94 & 0.93 & 0.85 & 0.88 & 0.77 & 0.70 \\
\hline MetaSVM & 629 & 59 & 1252 & 114 & 0.85 & 0.95 & 0.95 & 0.86 & 0.90 & 0.81 & 0.74 \\
\hline MutationAssessor & 278 & 36 & 999 & 96 & 0.74 & 0.97 & 0.96 & 0.79 & 0.85 & 0.73 & 0.64 \\
\hline MutationTaster2 & 694 & 251 & 1060 & 49 & 0.93 & 0.81 & 0.83 & 0.92 & 0.87 & 0.75 & 0.67 \\
\hline MutPred & 624 & 62 & 1240 & 117 & 0.84 & 0.95 & 0.95 & 0.86 & 0.90 & 0.80 & 0.73 \\
\hline PolyPhen HDIV & 668 & 213 & 925 & 37 & 0.95 & 0.81 & 0.84 & 0.94 & 0.88 & 0.77 & 0.69 \\
\hline PolyPhen HVAR & 633 & 151 & 1046 & 53 & 0.92 & 0.87 & 0.88 & 0.92 & 0.90 & 0.80 & 0.73 \\
\hline PON-P2 & 699 & 76 & 1235 & 48 & 0.94 & 0.94 & 0.90 & 0.96 & 0.94 & 0.87 & 0.82 \\
\hline PROVEAN & 646 & 263 & 1040 & 92 & 0.88 & 0.80 & 0.71 & 0.92 & 0.83 & 0.65 & 0.56 \\
\hline REVEL & 650 & 56 & 1255 & 93 & 0.87 & 0.96 & 0.95 & 0.88 & 0.92 & 0.83 & 0.77 \\
\hline SIFT & 659 & 330 & 979 & 84 & 0.89 & 0.75 & 0.78 & 0.87 & 0.82 & 0.64 & 0.55 \\
\hline VEST3 & 641 & 79 & 1232 & 102 & 0.86 & 0.94 & 0.93 & 0.87 & 0.90 & 0.80 & 0.73 \\
\hline
\end{tabular}




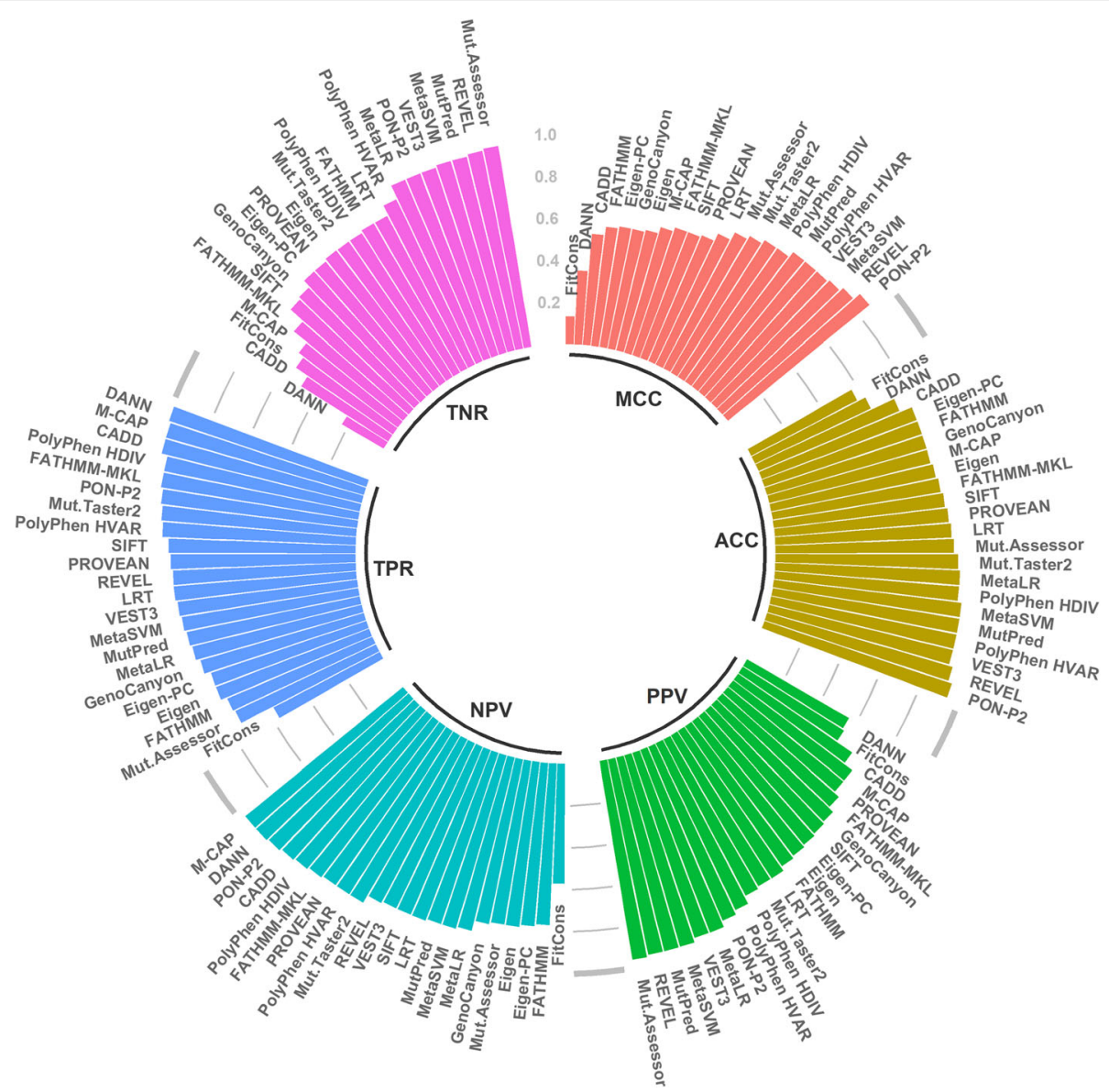

Fig. 1 Visualisation of six performance measures for tolerance predictors. The methods are organized according to their increasing performance for each of the measures

DANN and fitCons are the most unbalanced (Fig. 2). Venn diagram in Fig. 3 shows the numbers of correct predictions by the five best performing tools. The superior performance of PON-P2 originates from its capability alone or together with MetaSVM or MutPred to predict correct cases beyond what all the methods agree on.

The observed performance scores for the tools are close to the measures obtained when benchmarking with all kinds of proteins $[25-28,30]$. Although the ratio of membrane protein variants has been small during the training of many of the predictors, it does not show in the performances. This could be because despite MPs are embedded into lipid bilayers, only the surface of the transmembrane regions in MPs is in contact with lipids, for the other residues the environment is similar to internal positions in proteins or in protein complexes in general.

\section{Estimation of the sensitivity of MPs for variations}

Recently we estimated the sensitivity of nine groups of proteins for harmful variants based on PON-P2 predictions [31]. PON-P2 was found to have the best performance also for all the three regions in MPs (Table 6). As the results for all possible variants for this method were not available, we used a script to submit all 19 variants in every position in the MPs to the program in batches.

We created all the variants of the entire mpHTP $(5422$ proteins), a total of $56,686,557$ variants in 2,983,503 amino acids. The results are summarized in Table 8. In addition to overall sensitivity, we investigated whether the different regions of the TMPs had different sensitivities for variations. The variants were classified in three classes: neutral, pathogenic and those of unknown significance. $48.0 \%$ of the predicted positions in MPs were outside the membrane, $15.3 \%$ transmembrane, and $36.7 \%$ inside the membrane.

We obtained results for 5070 proteins $(93.5 \%$ of mpHTP entries) with high prediction coverage (95.5\%). There are several reasons for not reaching full coverage. Some proteins are unique for human, thus the required evolutionary information is missing and the predictor cannot work. Some sequences contained special characters, possibly for residue ambiguity. Those were not included to the analysis. Variations inside the membranes are predicted with slightly higher rate than transmembrane or outside the 
Table 7 Performance of tolerance predictors divided to membrane protein parts

\begin{tabular}{|c|c|c|c|c|c|c|c|c|c|c|c|c|c|c|c|c|c|c|c|c|c|}
\hline & \multicolumn{3}{|c|}{ Sensitivity } & \multicolumn{3}{|c|}{ Specificity } & \multicolumn{3}{|l|}{ PPV } & \multicolumn{3}{|l|}{ NPV } & \multicolumn{3}{|l|}{ ACC } & \multicolumn{3}{|l|}{ MCC } & \multicolumn{3}{|l|}{ OPM } \\
\hline & $\overline{\mathrm{TM}^{\mathrm{a}}}$ & $1^{a}$ & $\mathrm{O}^{\mathrm{a}}$ & $\overline{T M}$ & 1 & $\mathrm{O}$ & $\overline{\mathrm{TM}}$ & 1 & $\mathrm{O}$ & $\overline{T M}$ & I & $\mathrm{O}$ & $\overline{T M}$ & I & $\mathrm{O}$ & $\overline{T M}$ & I & $\mathrm{O}$ & $\overline{T M}$ & 1 & $\mathrm{O}$ \\
\hline CADD & 0.99 & 0.96 & 0.98 & 0.46 & 0.50 & 0.49 & 0.65 & 0.66 & 0.66 & 0.99 & 0.93 & 0.96 & 0.73 & 0.73 & 0.73 & 0.54 & 0.52 & 0.54 & 0.45 & 0.43 & 0.45 \\
\hline DANN & 00 & 0.99 & 0.99 & 0.20 & 0.24 & 0.24 & 0.56 & 0.57 & .56 & 1.00 & 0.97 & 0.95 & 0.60 & 0.62 & 0.61 & 0.33 & 0.35 & 0.34 & .30 & 0.31 & 0.30 \\
\hline igen & 0.78 & 0.89 & 0.70 & 0.73 & 0.83 & 0.82 & 0.74 & 0.84 & 0.79 & 0.77 & 0.88 & 0.73 & 0.76 & 0.86 & 0.76 & 0.51 & 0.71 & 0.52 & 0.43 & 0.63 & 0.44 \\
\hline gen & 0.78 & 0.88 & 0.70 & 0.74 & 0.80 & 0.81 & 0.75 & 0.82 & 0.79 & 0.77 & 0.87 & 0.73 & 0.76 & 0.84 & 0.76 & 0.52 & 0.69 & 0.51 & 0.44 & 0.60 & 0.43 \\
\hline EATHMM & 0.63 & 0.76 & 0.81 & 0.86 & 0.78 & 0.84 & 0.82 & 0.77 & 0.84 & 0.70 & 0.76 & 0.82 & 0.75 & 0.77 & 0.83 & 0.51 & 0.54 & 0.66 & 0.43 & 0.45 & 0.57 \\
\hline 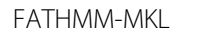 & 0 & .93 & 0 & 63 & 0.67 & 0.68 & 0.7 & 0.74 & 0.75 & 0.95 & 0.90 & 0.94 & 0.80 & 0.80 & 0.82 & 0.64 & 0.62 & 0.66 & 0.54 & 0.53 & 0.57 \\
\hline itCons & 0.44 & 0.69 & 0.55 & 0.72 & 0.55 & 0.53 & 0.61 & 0.60 & 0.54 & 0.56 & 0.64 & 0.54 & 0.58 & 0.62 & 0.54 & 0.17 & 0.24 & 0.08 & 0.20 & 0.24 & 0.16 \\
\hline eno & 0.78 & 84 & 0.81 & 0.72 & 0.77 & 0.77 & 0.74 & 0.78 & 0.78 & 0.76 & 0.83 & 0.80 & 0.75 & 0.81 & 0.79 & 0.50 & 0.61 & 0.58 & 0.42 & 0.52 & 0.49 \\
\hline RT & 0.94 & 0.85 & 0.85 & 0.75 & 0.82 & 0.83 & 0.79 & 0.83 & 0.83 & 0.93 & 0.85 & 0.85 & 0.85 & 0.84 & 0.84 & 0.70 & 0.67 & 0.68 & 0.62 & 0.59 & 0.59 \\
\hline M-CAP & 0.99 & 0.98 & 0.98 & 0.55 & 0.57 & 0.64 & 0.69 & 0.69 & 0.73 & 0.98 & 0.96 & 0.97 & 0.77 & 0.77 & 0.81 & 0.60 & 0.60 & 0.66 & 0.50 & 0.50 & 0.56 \\
\hline MetaLR & 0.81 & 0.81 & 0.85 & 0.92 & 0.93 & 0.96 & 0.91 & 0.92 & 0.95 & 0.83 & 0.83 & 0.87 & 0.86 & 0.87 & 0.91 & 0.73 & 0.74 & 0.82 & 0.65 & 0.66 & 0.75 \\
\hline 1etaSVM & 0.85 & 0.83 & 0.86 & 0.93 & 0.95 & 0.97 & 0.92 & 0.94 & 0.97 & 0.86 & 0.85 & 0.87 & 0.89 & 0.89 & 0.91 & 0.78 & 0.78 & 0.83 & 0.70 & 0.71 & 0.77 \\
\hline lutationAss & 0.89 & .60 & 0.78 & .92 & 0.98 & 0.97 & 0.91 & 0.97 & 96 & 0.89 & 0.71 & 0.82 & 90 & 79 & 0.88 & 80 & 0.63 & 0.77 & 73 & 0.53 & 69 \\
\hline MutationTaster2 & 0.99 & 0.92 & 0.91 & 0.78 & 0.81 & 0.82 & 0.82 & 0.83 & 0.83 & 0.99 & 0.91 & 0.90 & 0.89 & 0.87 & 0.86 & 0.79 & 0.74 & 0.73 & 0.71 & 0.65 & 0.65 \\
\hline arrted & 0.87 & 0.10 & 0.88 & 0.91 & 0.9 & (3) & 0.9 & 0. & S & 0.8 & 0.01 & 0.8 & 0.89 & 0.87 & 0.92 & 0.78 & 0.76 & 0.83 & 0.70 & 0.68 & 0 \\
\hline PolyPhen HDIV & 0.96 & 0.94 & 0.94 & 0.70 & 0.83 & 0.83 & 0.76 & 0.85 & 0.85 & 0.95 & 0.93 & 0.94 & 0.83 & 0.89 & 0.89 & 0.69 & 0.78 & 0.78 & 0.59 & 0.70 & 0.70 \\
\hline PolyPhen HVAR & 0 & 19 & 0 & 0.75 & 0.5 & 0.89 & 0.8 & 0.90 & 89 & 0.94 & 0.90 & 0.92 & 0.85 & 0.90 & 0.91 & 0.72 & 0.80 & 0.81 & 0.63 & 0.73 & $c$ \\
\hline PON-P2 & 0.94 & 0.93 & 0.94 & 0.90 & 0.94 & 0.96 & 0.90 & 0.88 & 0.92 & 0.94 & 0.96 & 0.97 & 0.92 & 0.93 & 0.95 & 0.85 & 0.85 & 0.89 & 0.78 & 0.80 & 0.85 \\
\hline PROVEAN & 0.91 & 0.83 & 0.89 & 0.70 & 0.81 & 0.81 & 0.75 & 0.82 & 0.83 & 0.89 & 0.83 & 0.88 & 0.81 & 0.82 & 0.85 & 0.63 & 0.65 & 0.71 & 0.54 & 0.56 & 0.62 \\
\hline REVEL & 0.90 & 0.85 & 0.88 & 0.93 & 0.96 & 0.97 & 0.93 & 0.95 & 0.96 & 0.91 & 0.86 & 0.89 & 0.92 & 0.90 & 0.92 & 0.84 & 0.81 & 0.85 & 0.77 & 0.74 & 0.79 \\
\hline SIFT & 0.94 & 0.86 & 0.88 & 0.65 & 0.74 & 0.78 & 0.73 & 0.77 & 0.80 & 0.91 & 0.84 & 0.87 & 0.80 & 0.80 & 0.83 & 0.62 & 0.60 & 0.67 & 0.52 & 0.52 & 0.58 \\
\hline VEST3 & 0.95 & 0.86 & 0.82 & 0.90 & 0.94 & 0.95 & 0.90 & 0.94 & 0.94 & 0.95 & 0.87 & 0.84 & 0.92 & 0.90 & 0.88 & 0.85 & 0.81 & 0.77 & 0.79 & 0.74 & 0.70 \\
\hline
\end{tabular}

${ }^{\mathrm{I}}$, inside the membrane; $\mathrm{O}$, outside the membrane; TM transmembrane

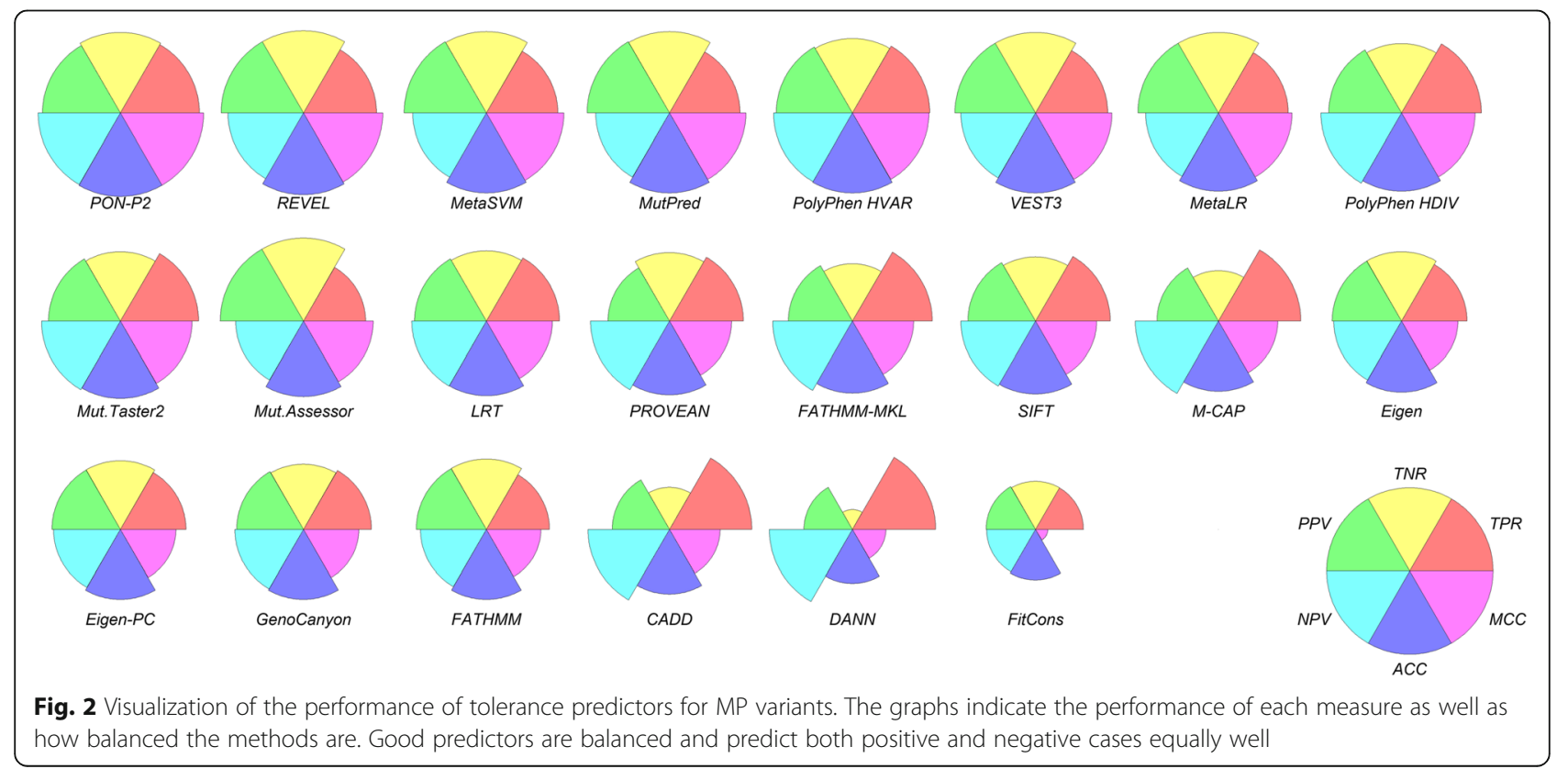




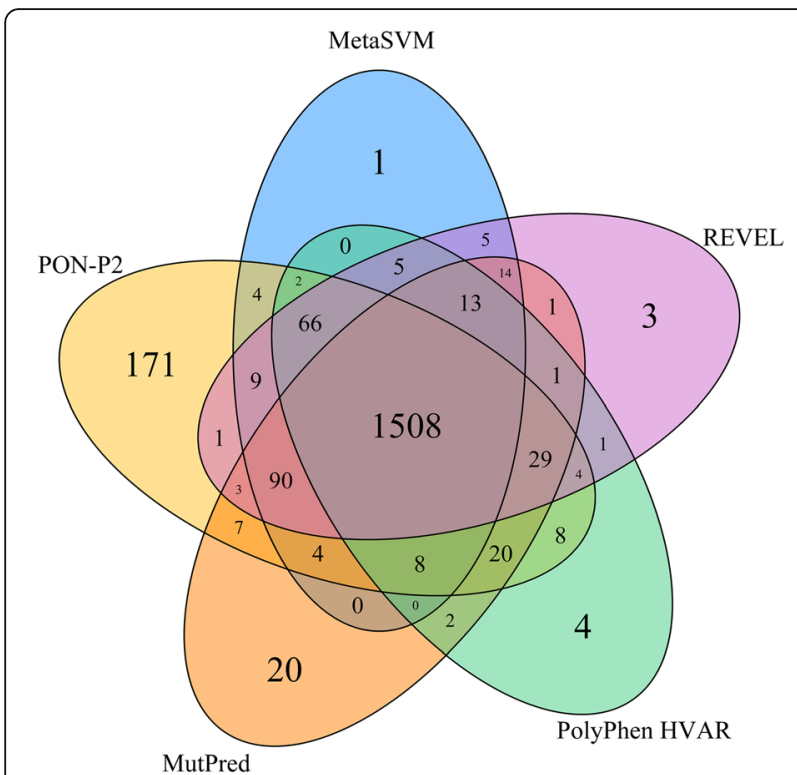

Fig. 3 Venn diagram for the congruence of the five best performing tools. The results are shown for 2002 variants that all the tools predicted. All the five methods predicted correct 1508 (75.3\%) variants membrane regions. The rate of unknown classifications is $41.66 \%$ and it is almost invariant for the three regions. This category contains many types of variants. The ratio is similar to many other proteins [31]. This category reflects also heterogeneity and continuum nature of pathogenicity [32]. The results are rather even for all the three regions in MPs. Transmembrane regions contain somewhat more likely pathogenic variants than the extramembranous regions: the ratio is 0.54 vs 0.41 and 0.48 . The share of neutral variants varies between 38.45 to $41.20 \%$. Totally close to 10 million variations were predicted to be pathogenic.

\section{Conclusion}

We tested the performance of two types of predictors on membrane proteins, their subcellular localization and variation interpretation in these proteins. We tested 22 variant tolerance predictors and 7 subcellular localization tools. The best variation prediction methods had similar prediction performance on transmembrane, inside and outside regions of transmembrane proteins and comparable to overall prediction performances for all types of proteins. The highest performing method was PON-P2, followed by REVEL, MetaSVM and VEST3. In the case of subcellular

Table 8 Statistics for predicted variants in human membrane proteome

\begin{tabular}{|c|c|c|c|c|}
\hline & Total & Outer & Transmembrane & Inner \\
\hline Number of proteins & 5422 & - & - & - \\
\hline Number of predicted proteins & 5070 & - & - & - \\
\hline Predicted proteins (\%) & 93.51 & - & - & - \\
\hline Number of amino acids & $2,983,503$ & $1,458,196$ & 456,186 & $1,069,121$ \\
\hline Number of predicted amino acids & $2,850,519$ & $1,367,843$ & 434,745 & $1,047,931$ \\
\hline Predicted amino acids (\%) & 95.54 & 93.80 & 95.30 & 98.02 \\
\hline Number of possible variants in all proteins/region & $56,686,557$ & $27,705,724$ & $8,667,534$ & $20,313,299$ \\
\hline Number of possible variants in predicted proteins/region & $54,159,861$ & $25,989,017$ & $8,260,155$ & $19,910,689$ \\
\hline Number of predicted variants & $53,310,412$ & $25,558,804$ & $8,169,606$ & $19,581,983$ \\
\hline Predicted variants (\% of possible) & 98.43 & 98.34 & 98.90 & 98.35 \\
\hline Number of variants predicted as neutral & $21,343,305$ & $10,529,555$ & $3,141,354$ & $7,672,377$ \\
\hline Neutral variants (\%) & 40.04 & 41.20 & 38.45 & 39.18 \\
\hline Average number of neutral variants per protein & 4197.31 & 2070.71 & 617.77 & 1508.83 \\
\hline Median number of neutral variants per protein & 2471 & 727 & 234 & 781 \\
\hline Number of variants predicted as pathogenic & $9,760,571$ & 4367,037 & $1,702,467$ & $3,691,067$ \\
\hline Pathogenic variants (\%) & 18.31 & 17.09 & 20.84 & 18.8 \\
\hline Average number of pathogenic variants per protein & 1919.48 & 858.81 & 334.80 & 725.87 \\
\hline Median number of pathogenic variants per protein & 173 & 19 & 2 & 20 \\
\hline Number of variants predicted as unknown & $22,206,536$ & $10,662,212$ & $3,325,785$ & $8,218,539$ \\
\hline Unknown variants (\%) & 41.66 & 41.72 & 40.71 & 41.97 \\
\hline Average number of unknown variants per protein & 4367.07 & 2096.80 & 654.04 & 1616.23 \\
\hline Median number of unknown variants per protein & 3013 & 714 & 290 & 616 \\
\hline Ratio of pathogenic and neutral variants & 0.46 & 0.41 & 0.54 & 0.48 \\
\hline
\end{tabular}


localization predictors we assessed separately the performance for single pass and multi pass membrane proteins. Predictions for multi pass proteins were more reliable than those for single pass proteins. Finally, we predicted the effects of all possible 19 substitutions in 5422 membrane proteins, a total of $56,686,557$ variants in 2,983,503 residues. Transmembrane regions seem to be somewhat more vulnerable for variations that regions inside and outside the membranes.

\section{Methods}

\section{Data for transmembrane proteins}

TMPs were obtained from two resources - Human Protein Atlas (HPA) and Human Transmembrane Proteome.

HPA [33-35] is a human proteome database based on quantitative transcriptomic analyses on tissue and organ levels. All major tissues and organs $(n=44)$ were analyzed by using 20,456 proprietary and 3572 externally obtained antibodies, totally providing more than 13 million immunochemistry images.

HPA database is organized in three main sections to tissue, cell and pathology atlases. Protein localization information was retrieved from the Cell Atlas section, where there are data for the subcellular localization of 12,073 proteins. The proteins are classified into 13 major organelles: actin filaments, centrosome, microtubules, intermediate filaments, cytosol, mitochondria, plasma membrane, vesicles, ER, Golgi apparatus, nuclear membrane, nucleoli, or nucleus. When using data from HPA, we considered as MPs proteins located to plasma membrane (including cell junctions) or nuclear membrane.

The reliability of data in HPA is indicated with four labels [34]. Location is validated, when it is according to one of the validation "pillars" proposed by an international working group [36]. Location is supported, when there is an agreement with the external experimental data from UniProtKB database. Location is approved, when external experimental information about the protein location is lacking, and location is uncertain, when there is contradictory information, such as with literature or transcriptomics data. HPA contains subcellular location data for 12,073 proteins [34].

HTP [1] is a database that combines experimental topology data together with predictions of the human transmembrane proteome. HTP is based on UniProt (UniRef 90) Human Proteome of 19,584 proteins. The proteins were filtered by using the Constrained Consensus Topology prediction method (CCTOP) [37] by using a consensus of ten major topology prediction methods. The current number of experimental and predicted MPs is 5423 (last updated August 7, 1917). The database contains topology information for each of the proteins.

\section{MP datasets}

Three datasets of TMPs were built to test predictors. They are available in VariBench [38] at [39].

\section{MP1289}

TMPs were filtered from the HPA location data as follows: the main location had to be one of the following: plasma membrane, cell junctions or nuclear membrane; the annotation for reliability score was validated, supported or approved; and the immunofluorescence (IF) location score of the three selected locations (plasma membrane, cell junction, nuclear membrane) was validated, supported or approved. Note that many proteins are localized to several compartments. The filtering yielded 1289 MPs. The sequences of these proteins were retrieved in fasta format from UniProt [10].

A negative set with the same number of proteins was obtained by picking randomly 1289 non-MP proteins by filtering out plasma membrane, cell-junction and nuclear membrane proteins from Cell Atlas. Since many MPs reside in several cellular organelles, we filtered out also proteins labelled to mitochondrion, ER, Golgi apparatus or vesicles. Further, we retrieved the "Subcellular location" information from UniProt for the proteins and filtered out proteins with membrane locations such as "cell membrane", "single-pass type II membrane protein" or "multi-pass membrane protein".

\section{MP508}

To obtain even more reliable experimentally validated dataset we excluded proteins annotated as approved from the MP1289 dataset and obtained 508 validated or supported proteins. As for the MP1289, a negative set was obtained by randomly choosing 508 non-MP proteins from Cell Atlas, excluding organelle proteins.

\section{mpHTP}

Since HPA database does not contain protein topology information, we obtained proteins with these details from HTP database. It contained topology information, experimental or predicted by very reliable CCTOP algorithm [37]. The topology is defined as I, M, O, L, S, T and $\mathrm{U}$ labels corresponding to cytoplasmic loops, membrane spanning segments, non-cytoplasmic loops, membrane re-entrant loops, signal peptides, transit peptides, and unknown regions, respectively [1]. In the entire mpHTP dataset of 5423 proteins, we found that two entries (HTP_152398 and HTP_010883) referred to the same protein with UniProt code P0DM63. Thus, the final number of entries in the mpHTP was 5422. By comparing the UniProt codes with DS1289 and DS508, we found that 450 and 205 proteins, respectively, were annotated in mpHTP. 
We retrieved a negative dataset by downloading nonTMPs from HTP and randomly selecting the same number as in the positive set (5422).

\section{Dataset for variants in MPs}

We could not find dedicated datasets for variants in MPs. Data from MutHTP [40] could not be used as many of the included cases are from cancers and without any evidence for disease. Therefore we collected a new dataset and mapped the locations of variants within the proteins. We used several sources. Non-disease related variants were obtained from Exome Aggregation Consortium (ExAC) dataset [41] among variants with a frequency higher than $1 \%$ but lower than $25 \%$ at least in one population [28]. Disease related membrane protein variants used to train PON-P2 [30] were obtained from VariBench. Additional cases were identified by searching locus specific variation databases (LSDBs) from LOVD for MPs identified in the first step. Additional databases were identified from Mutation Update articles in Human Mutation.

By combining and pruning the identified variants for duplicates and e.g. in proteins with unknown reliability score in HPA, we retrieved totally 2058 variants (MPvar) . There were 747 disease-related and 1311 benign cases.

\section{Subcellular localization prediction methods}

Subcellular localization prediction methods that allowed submission of large numbers of sequences were identified with literature and internet searches. The available and tested methods are described below.

\section{BUSCA}

The Bologna Unified Subcellular Component Annotator (BUSCA) [13] integrates prediction tools developed at the Bologna Biocomputing Group. It contains two types of predictors. There are methods for particular subsequence regions related to subcellular location, such as signal and transit peptides, GPI anchors and transmembrane domains. These methods include DeepSig [42], TPred3 [43], PredGPI [44], ENSEMBLE3.0 [45] and BetAware [46]. The second category includes methods predicting subcellular localization directly and includes BaCelLo [47], SChloro [48] and MemLoci [49].

They used two training subsets, Critical Assessment of Function Annotation 2 (CAFA2) data containing 2512 proteins from animals, 26 from fungi, 105 from plants, 87 from Gram-negative and 2 from Gram-positive bacteria; and CAFA3 data for 2559 proteins from animals, 535 from fungi, 489 from plants, 165 from Gramnegative and 16 from Gram-positive bacteria. The web server admits a maximum of 500 fasta sequences of up to 400,000 residues.

\section{Cello}

The subCELlular LOcalization (CELLO) predictor [14] is a multi-class support vector machine (SVM) classifier that uses a two-level system. In the first layer, the protein sequence is decomposed to extract four types of sequence coding schemes: amino acid composition, di-peptide composition, partitioned amino acid composition, and sequence composition based on the physicochemical properties of amino acids. Four independent SVM predictors were trained independently to generate probability distributions of the subcellular localizations. In the second layer the four schemes are combined to generate the final probability distribution of subcellular compartments and the localization with the highest probability.

For training, two datasets were used: 1444 proteins from Gram-negative bacteria distributed in five different subcellular compartments (extracellular, cytoplasmic, cytoplasmic membrane, periplasmic, outer membrane), and 7589 eukaryotic proteins distributed in 12 subcellular localizations (chloroplast, cytoplasmic, cytoskeleton, ER, extracellular, Golgi apparatus, lysosomal, mitochondrial, nuclear, peroxisomal, plasma membrane, vacuolar). Based on the input sequence and the cell type, CELLO returns a probability distribution of the subcellular localization and highlights the most probable one(s).

\section{DeepLoc1.0}

DeepLoc1.0 [15] is a deep learning predictor of recurrent neural networks (RNNs) with long-short-term memory (LSTM) cells, attention models and convolutional neural networks (CNNs). The CNNs extract short motifs from the sequence using 120 filters ( 20 for each of the sizes 1 , $3,5,9,15$ and 21 residues). The RNN scans the sequence in both directions using 256 LSTM units and has totally 1,000,512 dimensional output. The attention decoding layer uses an LSTM with 512 units through 10 decoding steps while the attention mechanism feedforward neural network (FFN) has 256 units. The final fully connected layer has one unit for membrane-bound and 10 units for other subcellular localizations [15].

DeepLoc training data was from UniProt and contained 13,858 proteins in 10 subcellular localizations (nucleus, cytoplasm, extracellular, mitochondrion, cell membrane, ER, plastid, Golgi apparatus, lysosome/vacuole, peroxisome). The web-server allows two types of predictions: accurate, for maximum of 50 sequences, and fast, for a maximum of 500 sequences.

\section{LocTree3}

LocTree3 [16] is an SVM approach to predict 18 eukaryotic subcellular localizations, 6 for bacteria and in 3 for Archaea. The SVMs are combined with homologybased inference by transferring localization annotations by homology through PSI-BLAST [50]. 
The training dataset contained three sub-datasets: 1682 eukaryotic proteins with 18 locations, 479 bacterial proteins with 6 locations, and 53 archaeal proteins with 3 locations. For each training set a different SVM was trained to build specific predictors. Web server and a standalone version are both available. We used the web server as it had no limitations for the number of submitted sequences.

\section{MultiLoc2}

MultiLoc2 [17] utilizes SVM predictor MultiLoc (Höglund et al. 2006), based on overall amino acids and the presence of known sorting signals, combined with phylogenetic profiles and Gene Ontology (GO) terms. MultiLoc2 integrates six subclassifiers: SVMTarget predicts localization categories based on N-terminal targeting sequences; SVMaac predicts localization based on overall amino acids composition; SVMSA predicts localization based on the presence of signal anchors; MotifSearch prediction is based on particular sequence motifs and structural domains; PhyloLoc utilizes information from homologous proteins in 78 fully sequenced genomes; and GOLoc takes benefit of GO annotations.

HighRes mode predicts 11 subcellular localizations (nuclear, cytoplasmic, mitochondrial, chloroplast, extracellular, plasma membrane, peroxisomal, ER, Golgi apparatus, lysosomal, vacuolar), whereas the LowRes is specialized for globular proteins and predicts 4 subcellular localizations (nuclear, cytoplasmic, mitochondrial, chloroplast). We used the HighRes mode.

MultiLoc2 training set contained 5959 sequences divided into 11 subcellular localizations. As the MultiLoc2 web server allows only 20 sequences per submission, we used a standalone version.

\section{SubCons}

SubCons [18] is based on ensemble approach, which combines the prediction results of CELLO2.5, LocTree2, MultiLoc2 and SherLoc2 using a Random Forest (RF) classifier. It classifies proteins into nine compartments (nucleus, cytoplasm/cytoskeleton, mitochondria, peroxisome, ER, Golgi apparatus, lysosome, plasma membrane, extracellular/secreted). SubCons returns a single subcellular localization result.

The training set contained 5484 proteins annotated in at least one experimental study. The SubCons web server has no sequence limit, so we used it. There is also a standalone version available.

\section{Wolf PSORT}

Wolf PSORT [19] is an extension of PSORT II [51] and uses PSORT [52] localization features to predict some features from iPSORT [53] along with amino acid composition. The features are used to convert amino acid sequences into numerical vectors, which are then classified with a weighted $k$-nearest neighbor classifier. Wolf PSORT classifies proteins into more than 10 localizations, including dual ones.

The training set was divided into fungi, plant and animal data containing 2113, 2333 and 12,771 proteins, respectively. The web server has no sequence input limits. Wolf PSORT returns the most probable localization with a number that roughly indicates the number of nearest neighbors to the query which localize to each site adjusted to account the possibility of dual localization.

\section{Variant tolerance predictors}

Large numbers of tools have been released for the prediction of pathogenicity or tolerance of amino acid substitutions. We tested the performance of 22 variant predictors on MP variants.

The methods included Combined Annotation Dependent Depletion (CADD) [54], Deleterious Annotation of genetic variants using Neural Networks (DANN) [55], Eigen [56], Eigen-PC [56], Functional Analysis through Hidden Markov Models (FATHMM) [57], FATHMM-MKL [58], fitCons [59], GenoCanyon [60], Likelihood Ratio Test (LRT) [61], Mendelian Clinically Applicable Pathogenicity (MCAP) [62], MetaLR [63], MetaSVM [63], MutationAssessor [64], MutationTaster2 [65], MutPred [66], Polymorphism Phenotyping v2 (PolyPhen) HDIV [67], PolyPhen HVAR [67], PON-P2 [30], Protein Variation Effect Analyzer (PROVEAN) [68], Rare Exome Variant Ensemble Learner (REVEL) [69], Sorting Intolerant From Tolerant (SIFT) [70], and Variant Effect Scoring Tool (VEST3) [71]. Variant effect predictions were downloaded from dbNSFP [72] apart for PON-P2, which were submitted via the program web site.

The tools can be classified based on how they have been implemented. Sequence information is the only feature in LRT, PROVEAN, and SIFT. These methods generate scoring data for sequence positions based on multiple sequence alignments of related sequences. Machine learning methods utilize various types of features for conservation, sequence characteristics, information about protein functions, propensities of the original and variant amino acids etc. Machine learning methods include CADD, DANN, Eigen, Eigen-PC, FATHMM, FATHHMM-MKL, GenoCanyon, M-CAP, MetaLR, MetaSVM, MutationAssessor, MutationTaster2, MutPred, PolyPhen both with HDIV and HVAR data, PON-P2, REVEL, and VEST. The machine learning-based methods are either unsupervised or supervised. The supervised methods have been trained on known disease-causing and benign variants. Algorithms in these tools include Bayesian, Hidden Markov Model (HMM), neural network (NN), RF, SVM and other approaches. fitCons is based on clustering of functional genomic fingerprints and fitness calculations. 
CADD ranks single nucleotide variants (SNVs) and short insertions and deletions. It assumes two types of variants: the proxy-neutral variants, fixed by purifying selection, and proxy-deleterious variants, from de novo variations free of positive selection. DANN is a deep neural network approach trained with about 30 millions of variants. It is very close to CADD.

Eigen is an unsupervised spectral approach. Its main assumption is that the variants can be partioned in two distinct groups: functional and non-functional. A weighted linear combination of annotations is constructed, based on these estimated accuracies. Eigen-PC is conceptually simpler, based on eigen decomposition of the annotation covariance matrix. It uses the lead eigenvector to weight the individual annotations.

FATHMM is species-independent but with optional species-specific weightings. It creates an Hidden Markov Model based multiple sequence alignment and protein domain analysis. It derives weights from the relative frequencies of disease-associated and functionally neutral amino acid substitutions mapped onto conserved protein domains. FATHMM-MKL is a SVM tool that in addition to substitutions predicted effects of insertions and deletions.

fitCons estimates variation fitness consequences according to functional genomic fingerprints by integrating evolutionary and functional data.GenoCanyon is an unsupervised statistical learning method. It provides a posteriori probabilities for functional genomic positions and are used as deleteriousness proxies.

LRT is a statistical method based on calculation of likelihood ratio by using a comparative genomic data set for 32 vertebrates. Variants at conserved positions are considered as likely deleterious. LRT compares the probability of the data under a conserved model relative to a neutral model.

M-CAP is a pathogenicity likelihood score calculated with gradient boosting trees based on a number of features for sequences and their conservation in 99 primate, mammalian, and vertebrate genomes.

MetaSVM and MetaLR are metapredictors, i.e. combine predictions from other tools by using SVM and logistic regression (LR) algorithms, respectively. MutationAssessor uses evolutionary information coming from clustered MSAs of homologous sequences in subfamilies to analyze functional specificity on the background of conservation of overall function. Entropy function of the residue distribution is used as a measure of conservation and as an estimate for the impact of variants.

MutationTaster analyses evolutionary conservation, splice-site changes, loss of protein features and changes that might affect the amount of mRNA. MutPred utilizes SVM and RF methods for calculating the posterior probability that a residue has a certain structural or functional property and probability for the loss or gain of the property.
PolyPhen-2 uses eight sequence-based and three structure-based predictive features. The difference between HDIV and HVAR versions is the training sets. The former has been trained with Mendelian diseasecausing variants annotated in the UniProt and affecting protein stability or function. HVAR version is trained on all the human disease-causing variants in UniProt, while SNVs without annotated involvement in disease are considered as benign. HDIV version is more suited to evaluate rare alleles at loci in complex phenotypes and HVAR in Mendelian diseases.

PON-P2 utilizes RF algorithm. Feature selection of 622 characteristics indicated that only 8 are essential for the predictor. PON-P2 utilizes information about evolutionary conservation, physical and biochemical properties of amino acids and GO annotations. PROVEAN uses an alignment score (delta score) as stability index. The larger the introduced difference to the score due to variation the more damaging is the variant. The tool collects a set of homologous proteins of the query protein and compute the delta score for each pairwise alignment.

REVEL is a RF-classifier based meta-predictor that uses results from MutPred, FATHMM, VEST, PolyPhen, SIFT, PROVEAN, MutationAssessor, MutationTaster, LRT, GERP, SiPhy, phyloP, and phastCons. The concept of SIFT is similar to REVEL. Evolutionary information is revealed from MSA and used to predict how tolerated the variant is. VEST is a RF-tool that prioritizes amino acid substitutions that alter protein activity.

\section{Scoring indices}

In order to assess the performance of predictors we calculated six measures according to published guidelines $[73,74]$. These measures are based on confusion/contingency matrix in which the actual conditions are compared with the prediction outcomes and data items are grouped as true positives (TP), true negatives (TN), false positives (FP) and false negatives (FN). Since the numbers of positive and negative cases in the datasets were not equal, the numbers were normalized to calculate the following measures.

The sensitivity or true positive rate (TPR) is the rate of TP over the total of positive conditions

$$
\text { Sensitivity }=\frac{T P}{T P+F N} .
$$

The specificity or true negative rate (TNR) is the rate of TP over the total of negative conditions

$$
\text { Specificity }=\frac{T N}{F P+T N} .
$$

The positive predictive value (PPV) or precision is the rate of true positive results over the total positive prediction 


$$
P P V=\frac{T P}{T P+F P} .
$$

The negative predictive value (NPV) is the rate of true negative results over the total negative prediction:

$$
N P V=\frac{T N}{T N+F N} .
$$

The accuracy (ACC) is the rate of correctly predicted conditions over the total observations

$$
\text { Accuracy }=\frac{T P+T N}{T P+T N+F P+F N} .
$$

The Matthews Correlation Coefficient (MCC) is a robust measure. It takes into account both over and under prediction and it returns a value from -1 to $+1 .+1$ identifies a perfect prediction, 0 identifies random prediction, and -1 means a totally inverse prediction

$$
M C C=\frac{T P \times T N-F P \times F N}{\sqrt{(T P+F P)(T P+F N)(T N+F P)(T N+F N)}} .
$$

The Overall Perfomance Measure (OPM) is a performance index used to visualize all the previous six indices simultaneously. OPM is represented by normalized volume of the performance cuboid, which ranges from 0 to 1 [30], where $\mathrm{nMCC}$ is calculated by rescaling the MCC value from 0 to 1 :

$$
O P M=\frac{(P P V+N P V)(\text { Sensitivity }+ \text { Specificity })\left(\text { Accuracy }+\left(\frac{1+M C C}{2}\right)\right)}{8}
$$

\section{Additional file}

Additional file 1: Table S1. Numbers of proteins in membrane subcellular localizations. Table S2. Performance of subcellular localization predictors on MP1289 restricted to one subcellular localization per protein. Table S3. Performance of subcellular localization predictors on single and multi pass membrane proteins. (DOCX $21 \mathrm{~kb}$ )

\section{Abbreviations}

ACC: accuracy; CAFA: Critical assessment of function annotation; CNN: Convolutional neural network; EC: Enzyme commission;

ER: Endoplasmic reticulum; ExAC: Exome aggregation consortium; FFN: Feed forward network; FN: False negative; FP: False positive; GO: Gene ontology; GPI: Glycosylphosphatidylinositol; HMM: Hidden Markov model; HPA: Human protein atlas; HTP: Human transmembrane proteome; LSTM: Long-short-term memory; MCC: Matthews correlation coefficient; ML: Machine learning; MLP: Multi location protein; MP: Membrane protein; MP1289: Dataset for membrane proteins; MP508: Dataset for membrane proteins; mpHTP: Dataset for membrane proteins; NN: Neural network; NPV: Negative predictive value; OPM: Overall performance measure; PDB: Protein data bank; PPV: Positive predictive value; RF: Random forest; RNN: Recurrent neural network; SVM: Support vector machine; TM: Transmembrane; TMP: Transmembrane protein; TN: True negative; TNR: True negative rate; TP: True positive; TPR: True positive rate

Acknowledgements

Not applicable.

\section{About this supplement}

This article has been published as part of BMC Genomics Volume 20 Supplement 8, 2019: Proceedings of Varl-COSI 2018: identification and annotation of genetic variants in the context of structure, function, and disease: genomics. The full contents of the supplement are available online at https:// bmcgenomics.biomedcentral.com/articles/supplements/volume-20supplement-8.

\section{Authors' contributions}

TO collected the datasets, performed analyses, interpreted data and drafted the manuscript. MV conceived the idea, interpreted data, supervised the project and drafted the manuscript. All authors read and approved the final manuscript.

\section{Funding}

Financial support from the Swedish Research Council and Erasmus+ is gratefully acknowledged. Publication costs are funded by Swedish Research Council. Funding bodies had no role in the design of the study and collection, analysis, and interpretation of data and in writing the manuscript.

\section{Availability of data and materials}

The datasets generated and analyzed during the current study are available in the VariBench repository [75]

Ethics approval and consent to participate

Not applicable.

\section{Consent for publication}

Not applicable.

\section{Competing interests}

The authors declare that they have no competing interests.

\section{Author details}

${ }^{1}$ International Master in Bioinformatics, School of Science, University of Bologna, Bologna, Italy. ${ }^{2}$ Department of Experimental Medical Science, BMC B13, Lund University, SE-22184 Lund, Sweden.

Published: 16 July 2019

\section{References}

1. Dobson L, Remenyi I, Tusnady GE. The human transmembrane proteome. Biol Direct. 2015:10:31.

2. Yildirim MA, Goh Kl, Cusick ME, Barabasi AL, Vidal M. Drug-target network. Nat Biotechnol. 2007:25:1119-26.

3. Bakheet TM, Doig AJ. Properties and identification of human protein drug targets. Bioinformatics. 2009:25:451-7.

4. Chou KC, Elrod DW. Prediction of membrane protein types and subcellular locations. Proteins. 1999:34:137-53.

5. Sällman Almén MS, Nordström KJ, Fredriksson R, Schiöth HB. Mapping the human membrane proteome: a majority of the human membrane proteins can be classified according to function and evolutionary origin. BMC Biol. 2009;7:50.

6. Bowie JU. Helix packing in membrane proteins. J Mol Biol. 1997;272:780-9.

7. Lomize MA, Pogozheva ID, Joo H, Mosberg HI, Lomize AL. OPM database and PPM web server: resources for positioning of proteins in membranes. Nucleic Acids Res. 2012;40:D370-6.

8. Tusnady GE, Kalmar L, Simon I. TOPDB: topology data bank of transmembrane proteins. Nucleic Acids Res. 2008;36:D234-9.

9. Berman HM, Westbrook J, Feng Z, Gilliland G, Bhat TN, Weissig H, Shindyalov IN, Bourne PE. The Protein Data Bank. Nucleic Acids Res. 2000;28:235-42

10. UniProt Consortium T. UniProt: the universal protein knowledgebase. Nucleic Acids Res. 2018;46:2699.

11. Saier MH Jr, Reddy VS, Tsu BV, Ahmed MS, Li C, Moreno-Hagelsieb G. The transporter classification database (TCDB): recent advances. Nucleic Acids Res. 2016:44:D372-9.

12. Committee IUoBaMBN, Webb EC: Enzyme nomenclature 1992: recommendations of the Nomenclature Committee of the International Union of Biochemistry and Molecular Biology on the nomenclature and 
Classification of enzymes. In. San Diego: Published for the International Union of Biochemistry and Molecular Biology by Academic Press; 1992.

13. Savojardo C, Martelli PL, Fariselli P, Profiti G, Casadio R. BUSCA: an integrative web server to predict subcellular localization of proteins. Nucleic Acids Res. 2018;46: W459-w466.

14. Yu CS, Chen YC, Lu CH, Hwang JK. Prediction of protein subcellular localization. Proteins. 2006;64:643-51.

15. Almagro Armenteros JJ, Sonderby CK, Sonderby SK, Nielsen H, Winther O. DeepLoc: prediction of protein subcellular localization using deep learning Bioinformatics. 2017;33:3387-95.

16. Goldberg T, Hecht M, Hamp T, Karl T, Yachdav G, Ahmed N, Altermann U, Angerer P, Ansorge S, Balasz K, et al. LocTree3 prediction of localization. Nucleic Acids Res. 2014:42:W350-5.

17. Blum T, Briesemeister S, Kohlbacher O. MultiLoc2: integrating phylogeny and gene ontology terms improves subcellular protein localization prediction. BMC Bioinformatics. 2009;10:274.

18. Salvatore M, Warholm P, Shu N, Basile W, Elofsson A. SubCons: a new ensemble method for improved human subcellular localization predictions. Bioinformatics. 2017:33:2464-70.

19. Horton P, Park KJ, Obayashi T, Fujita N, Harada H, Adams-Collier CJ, Nakai K. WoLF PSORT: protein localization predictor. Nucleic Acids Res. 2007;35:W585-7.

20. Pierleoni A, Indio V, Savojardo C, Fariselli P, Martelli PL, Casadio R. MemPype: a pipeline for the annotation of eukaryotic membrane proteins. Nucleic Acids Res. 2011;39:W375-80.

21. Garg A, Bhasin M, Raghava GP. Support vector machine-based method for subcellular localization of human proteins using amino acid compositions, their order, and similarity search. J Biol Chem. 2005;280:14427-32.

22. Chou KC, Wu ZC, Xiao X. iLoc-hum: using the accumulation-label scale to predict subcellular locations of human proteins with both single and multiple sites. Mol BioSyst. 2012;8:629-41.

23. Shatkay $H$, Hoglund A, Brady S, Blum T, Donnes P, Kohlbacher O. SherLoc: high-accuracy prediction of protein subcellular localization by integrating text and protein sequence data. Bioinformatics. 2007;23:1410-7.

24. Altschul SF, Gish W, Miller W, Myers EW, Lipman DJ. Basic local alignment search tool. J Mol Biol. 1990;215:403-10.

25. Bendl J, Stourac J, Salanda O, Pavelka A, Wieben ED, Zendulka J, Brezovsky J, Damborsky J. PredictSNP: robust and accurate consensus classifier for prediction of disease-related mutations. PLoS Comput Biol. 2014;10: e1003440.

26. Riera C, Padilla N, de la Cruz X. The complementarity between proteinspecific and general pathogenicity predictors for amino acid substitutions. Hum Mutat. 2016;37:1012-24.

27. Thusberg J, Olatubosun A, Vihinen M. Performance of mutation pathogenicity prediction methods on missense variants. Hum Mutat. 2011 32:358-68.

28. Niroula A, Vihinen M. How good are pathogenicity predictors in detecting benign variants? PLoS Comput Biol. 2019;15:e1006481.

29. Niroula A, Vihinen M. Variation interpretation predictors: principles, types, performance, and choice. Hum Mutat. 2016:37:579-97.

30. Niroula A, Urolagin S, Vihinen M. PON-P2: prediction method for fast and reliable identification of harmful variants. PLoS One. 2015;10(2):e0117380.

31. Schaafsma GCP, Vihinen M. Large differences in proportions of harmful and benign amino acid substitutions between proteins and diseases. Hum Mutat. 2017;38:839-48.

32. Vihinen M. How to define pathogenicity, health, and disease? Hum Mutat 2017;38:129-36

33. Uhlen M, Fagerberg L, Hallstrom BM, Lindskog C, Oksvold P, Mardinoglu A Sivertsson A, Kampf C, Sjostedt E, Asplund A, et al. Proteomics Tissue-based map of the human proteome. Science. 2015;347:1260419.

34. Thul PJ, Akesson L, Wiking M, Mahdessian D, Geladaki A, Ait Blal H, Alm T, Asplund A, Bjork L, Breckels LM, et al. A subcellular map of the human proteome. Science. 2017;356:820

35. Uhlen M, Bjorling E, Agaton C, Szigyarto CA, Amini B, Andersen E, Andersson AC, Angelidou P, Asplund A, Asplund C, et al. A human protein atlas for normal and cancer tissues based on antibody proteomics. Mol Cell Proteomics. 2005:4:1920-32.

36. Uhlen M, Bandrowski A, Carr S, Edwards A, Ellenberg J, Lundberg E, Rimm DL, Rodriguez H, Hiltke T, Snyder M, et al. A proposal for validation of antibodies. Nat Methods. 2016;13:823-7.

37. Dobson L, Remenyi I, Tusnady GE. CCTOP: a consensus constrained TOPology prediction web server. Nucleic Acids Res. 2015;43:W408-12.
38. Nair PS, Vihinen M. VariBench: a benchmark database for variations. Hum Mutat. 2013:34:42-9.

39. Membrane protein benchmark datasets. http://structure.bmc.lu.se/ VariBench/strucmapall.php. Accessed 12 Mar 2019.

40. Kulandaisamy A, Binny Priya S, Sakthivel R, Tarnovskaya S, Bizin I, Honigschmid P, Frishman D, Gromiha MM. MutHTP: mutations in human transmembrane proteins. Bioinformatics. 2018;34:2325-6.

41. Lek M, Karczewski KJ, Minikel EV, Samocha KE, Banks E, Fennell T, O'DonnellLuria AH, Ware JS, Hill AJ, Cummings BB, et al. Analysis of protein-coding genetic variation in 60,706 humans. Nature. 2016;536:285-91.

42. Savojardo C, Martelli PL, Fariselli P, Casadio R. DeepSig: deep learning improves signal peptide detection in proteins. Bioinformatics. 2018;34: 1690-6.

43. Savojardo C, Martelli PL, Fariselli P, Casadio R. TPpred3 detects and discriminates mitochondrial and chloroplastic targeting peptides in eukaryotic proteins. Bioinformatics. 2015;31:3269-75

44. Pierleoni A, Martelli PL, Casadio R. PredGPI: a GPI-anchor predictor. BMC Bioinformatics. 2008;9:392.

45. Martelli PL, Fariselli P, Casadio R. An ENSEMBLE machine learning approach for the prediction of all-alpha membrane proteins. Bioinformatics. 2003; 19(Suppl 1):i205-11.

46. Savojardo C, Fariselli P, Casadio R. BETAWARE: a machine-learning tool to detect and predict transmembrane beta-barrel proteins in prokaryotes. Bioinformatics. 2013;29:504-5.

47. Pierleoni A, Martelli PL, Fariselli P, Casadio R. BaCelLo: a balanced subcellular localization predictor. Bioinformatics. 2006;22:e408-16.

48. Savojardo C, Martelli PL, Fariselli P, Casadio R. SChloro: directing Viridiplantae proteins to six chloroplastic sub-compartments. Bioinformatics. 2017;33:347-53.

49. Pierleoni A, Martelli PL, Casadio R. MemLoci: predicting subcellular localization of membrane proteins in eukaryotes. Bioinformatics. 2011;27: 1224-30.

50. Altschul SF, Madden TL, Schaffer AA, Zhang J, Zhang Z, Miller W, Lipman DJ, Gapped BLAST. PSI-BLAST: a new generation of protein database search programs. Nucleic Acids Res. 1997;25:3389-402.

51. Horton P, Nakai K. Better prediction of protein cellular localization sites with the $k$ nearest neighbors classifier. Proc Int Conf Intell Syst Mol Biol. 1997:5:147-52.

52. Nakai K, Kanehisa M. A knowledge base for predicting protein localization sites in eukaryotic cells. Genomics. 1992:14:897-911.

53. Bannai H, Tamada $Y$, Maruyama O, Nakai K, Miyano S. Extensive feature detection of $\mathrm{N}$-terminal protein sorting signals. Bioinformatics. 2002;18: 298-305.

54. Kircher M, Witten DM, Jain P, O'Roak BJ, Cooper GM. A general framework for estimating the relative pathogenicity of human genetic variants. Nat Genet. 2014;46:310-5.

55. Quang D, Chen Y, Xie X. DANN: a deep learning approach for annotating the pathogenicity of genetic variants. Bioinformatics. 2015;31:761-3.

56. Ionita-Laza I, McCallum K, Xu B, Buxbaum JD. A spectral approach integrating functional genomic annotations for coding and noncoding variants. Nat Genet. 2016:48:214-20.

57. Shihab HA, Gough J, Cooper DN, Stenson PD, Barker GL, Edwards KJ, Day IN, Gaunt TR. Predicting the functional, molecular, and phenotypic consequences of amino acid substitutions using hidden Markov models. Hum Mutat. 2013:34:57-65.

58. Shihab HA, Rogers MF, Gough J, Mort M, Cooper DN, Day IN, Gaunt TR, Campbell C. An integrative approach to predicting the functional effects of non-coding and coding sequence variation. Bioinformatics. 2015;31:1536-43.

59. Gulko B, Hubisz MJ, Gronau I, Siepel A. A method for calculating probabilities of fitness consequences for point mutations across the human genome. Nat Genet. 2015;47:276-83.

60. Lu Q, Hu Y, Sun J, Cheng Y, Cheung KH, Zhao H. A statistical framework to predict functional non-coding regions in the human genome through integrated analysis of annotation data. Sci Rep. 2015;5:10576.

61. Chun S, Fay JC. Identification of deleterious mutations within three human genomes. Genome Res. 2009;19:1553-61.

62. Jagadeesh KA, Wenger AM, Berger MJ, Guturu H, Stenson PD, Cooper DN, Bernstein JA, Bejerano G. M-CAP eliminates a majority of variants of uncertain significance in clinical exomes at high sensitivity. Nat Genet. 2016; 48:1581-6. 
63. Dong C, Wei P, Jian X, Gibbs R, Boerwinkle E, Wang K, Liu X. Comparison and integration of deleteriousness prediction methods for nonsynonymous SNVs in whole exome sequencing studies. Hum Mol Genet. 2015;24:2125-37.

64. Reva B, Antipin Y, Sander C. Predicting the functional impact of protein mutations: application to cancer genomics. Nucleic Acids Res. 2011;39:e118.

65. Schwarz JM, Cooper DN, Schuelke M, Seelow D. MutationTaster2: mutation prediction for the deep-sequencing age. Nat Methods. 2014;11:361-2.

66. Li B, Krishnan VG, Mort ME, Xin F, Kamati KK, Cooper DN, Mooney SD, Radivojac P. Automated inference of molecular mechanisms of disease from amino acid substitutions. Bioinformatics. 2009:25:2744-50.

67. Adzhubei IA, Schmidt S, Peshkin L, Ramensky VE, Gerasimova A, Bork P, Kondrashov AS, Sunyaev SR. A method and server for predicting damaging missense mutations. Nat Methods. 2010;7:248-9.

68. Choi Y, Sims GE, Murphy S, Miller JR, Chan AP. Predicting the functional effect of amino acid substitutions and indels. PLoS One. 2012;7:e46688.

69. Ioannidis NM, Rothstein JH, Pejaver V, Middha S, McDonnell SK, Baheti S, Musolf A, Li Q, Holzinger E, Karyadi D, et al. REVEL: an ensemble method for predicting the pathogenicity of rare missense variants. Am J Hum Genet. 2016;99:877-85

70. Ng PC, Henikoff S. Predicting deleterious amino acid substitutions. Genome Res. 2001:11:863-74.

71. Carter H, Douville C, Stenson PD, Cooper DN, Karchin R. Identifying Mendelian disease genes with the variant effect scoring tool. BMC Genomics. 2013;14(Suppl 3):S3.

72. Liu X, Jian X, Boerwinkle E. dbNSFP: a lightweight database of human nonsynonymous SNPS and their functional predictions. Hum Mutat. 2011;32:894-9.

73. Vihinen M. How to evaluate performance of prediction methods? Measures and their interpretation in variation effect analysis. BMC Genomics. 2012; 13(Suppl 4):S2

74. Vihinen M. Guidelines for reporting and using prediction tools for genetic variation analysis. Hum Mutat. 2013;34:275-82.

75. Membrane proteins. http://structure.bmc.lu.se/NariBench/strucmapall.php. Accessed 12 March 2019

\section{Publisher's Note}

Springer Nature remains neutral with regard to jurisdictional claims in published maps and institutional affiliations.

Ready to submit your research? Choose BMC and benefit from:

- fast, convenient online submission

- thorough peer review by experienced researchers in your field

- rapid publication on acceptance

- support for research data, including large and complex data types

- gold Open Access which fosters wider collaboration and increased citations

- maximum visibility for your research: over $100 \mathrm{M}$ website views per year

At $\mathrm{BMC}$, research is always in progress.

Learn more biomedcentral.com/submissions 